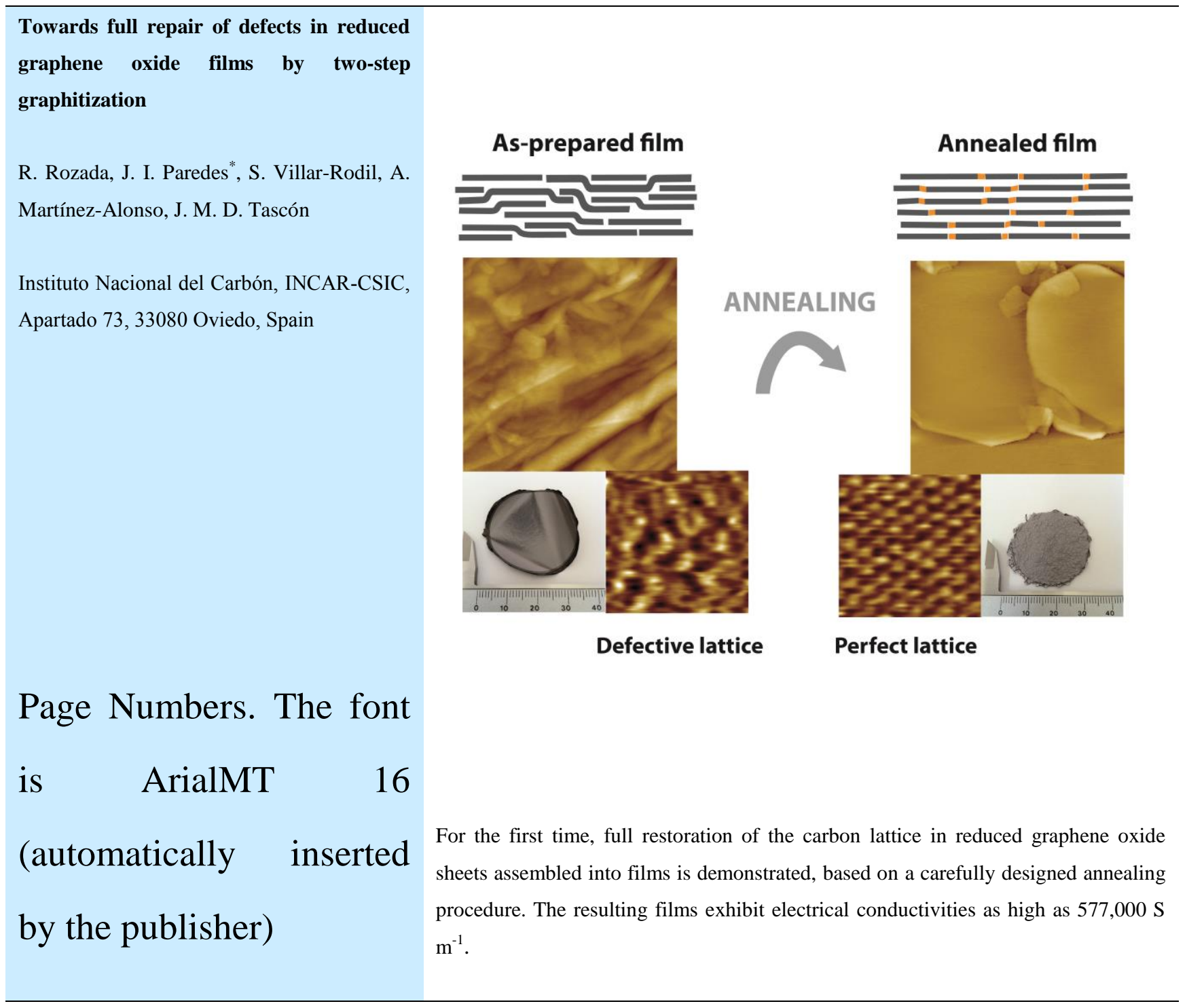




\title{
Towards Full Repair of Defects in Reduced Graphene Oxide Films by Two-step Graphitization
}

\author{
R. Rozada, J.I. Paredes( $\square \bowtie)$, S. Villar-Rodil, A. Martínez-Alonso, J.M.D. Tascón \\ Instituto Nacional del Carbón, INCAR-CSIC, Apartado 73, 33080 Oviedo, Spain \\ Received: day month year / Revised: day month year / Accepted: day month year (automatically inserted by the publisher) \\ (C) Tsinghua University Press and Springer-Verlag Berlin Heidelberg 2011
}

\begin{abstract}
The complete restoration of a perfect carbon lattice has been a central issue in the research on graphene derived from graphite oxide since this preparation route was first proposed several years ago, but such a goal has so far remained elusive. Here, we demonstrate that the highly defective structure of reduced graphene oxide sheets assembled into free-standing, paper-like films can be fully repaired by means of high temperature annealing (graphitization). Characterization of the films by X-ray photoelectron and Raman spectroscopy, X-ray diffraction and scanning tunneling microscopy indicated that the main stages in the transformation of the films were (i) complete removal of oxygen functional groups and generation of atomic vacancies (up to $1500{ }^{\circ} \mathrm{C}$ ), and (ii) vacancy annihilation and coalescence of adjacent overlapping sheets to yield continuous polycrystalline layers $\left(1800-2700{ }^{\circ} \mathrm{C}\right)$ similar to those of highly oriented graphites. The prevailing type of defect in the polycrystalline layers were the grain boundaries separating neighboring domains, which were typically a few hundred nanometers in lateral size, exhibited long-range graphitic order and were virtually free of even atomic-sized defects. The electrical conductivity of the annealed films was as high as $577,000 \mathrm{~S} \mathrm{~m}^{-1}$, which is by far the largest value reported to date for any material derived from graphene oxide, and strategies for further improvement without the need to resort to higher annealing temperatures are suggested. Overall, the present work opens the prospect of truly achieving a complete restoration of the carbon lattice in graphene oxide materials.
\end{abstract}

\section{KEYWORDS}

Graphene; Graphene oxide; Films; Annealing; Defect

\section{Introduction}

The last decade has witnessed the rapid emergence of graphene research in a remarkable variety of fronts. Research activities have covered basic science aspects of this two-dimensional carbon material, to provide fundamental insight into its electronic, mechanical, thermal and optical properties [1,2], as well as the exploration of its potential towards different technologically relevant applications, for instance in electronics [3], energy conversion and storage [4], chemical and biological sensing [5,6], catalysis [7] or biomedicine [8]. The development of versatile and cost-effective methodologies for the mass production and

Address correspondence to J. I. Paredes, paredes@incar.csic.es 
processing of graphene has been and continues to be central to many of these efforts [9-11]. In this regard, one approach that has proved particularly fruitful is the so-called graphite oxide route, which is based on the exfoliation and reduction of this strongly oxygenated derivative of graphite to afford graphene sheets in large quantities [9,12-17]. In addition to its massively scalable production, this type of graphene (usually referred to as reduced graphene oxide) exhibits several attractive features that are important from a practical viewpoint, including the fact that it is readily dispersible in aqueous and organic medium and its versatility for chemical functionalization and combination with other materials [11,12,18].

On the other hand, the carbon lattice in reduced graphene oxide is well known to contain a large number of structural defects and distortions. Such distortions are mainly introduced as a result of the grafting of oxygen functional groups (e.g., hydroxyls and epoxides) during the conversion of graphite to graphite oxide [19]. Although the subsequent reduction step is intended to remove these functional groups and thus restore the originally pristine graphene lattice, virtually all the strategies that have so far been put to practice to this end have yielded products with a significant amount of residual oxygen-containing groups and defects, which are apparently very difficult to eliminate $[14,15,19]$. This point has been verified both directly by high resolution microscopy imaging of reduced graphene oxide sheets $[20,21]$ and indirectly through the observation of a relatively poor recovery of highly defect-sensitive properties, such as the electrical conductivity $[22,23]$.

To improve the structural quality and properties of graphite oxide-derived graphene sheets, and ultimately the performance of devices and materials obtained thereof, several groups have implemented defect repair techniques based on the supply of carbon atoms from a hydrocarbon gas source at $\sim 600-900{ }^{\circ} \mathrm{C}$, i.e. by chemical vapour deposition (CVD) [24-26]. Even though the repaired sheets displayed significantly increased values of electrical conductivity, the actual degree of defect healing attained by this method was very far from complete. Furthermore, because the healing of subsurface defects does not appear to be possible [27], the CVD technique is only suited for the repair of monolayer, but not multilayer, graphene oxide/reduced graphene oxide films. Building upon the pioneering work on the heat treatment of graphite oxide reported some decades ago [28-33], the thermal annealing of graphene oxide up to moderately high temperatures $\left(\sim 1100{ }^{\circ} \mathrm{C}\right)$ has also been extensively investigated in recent years $[14,15,23,34-36]$, but the amount of residual oxygen and structural defects remaining on such thermally reduced graphene is generally comparable to that achieved with some of the most effective chemical reduction approaches carried out at much lower temperatures $\left(<100{ }^{\circ} \mathrm{C}\right)$ $[14,15]$, i.e. it is still rather large. Thus, in the absence of other alternatives, it can be postulated that a full repair of defects in graphene oxide or reduced graphene oxide will be most realistically accomplished under graphitization conditions, which involve very high annealing temperatures (e.g., $2000{ }^{\circ} \mathrm{C}$ or above) [37]. Some very recent work along this line suggests that this might indeed be the case $[38,39]$, but a thorough investigation on the topic (for example, encompassing a wide range of graphitization temperatures) has not yet been reported. Furthermore, complete removal of defects from the sheets has not been demonstrated and the details of their structural evolution down to the nanometer/atomic scales have remained unknown.

Here, we present a comprehensive study on the structural, chemical and electrical characteristics of free-standing, paper-like films of chemically reduced graphene oxide (reduced graphene oxide paper) that have been subjected to graphitization treatments at different temperatures, from 1500 up to $2700{ }^{\circ} \mathrm{C}$. Reduced graphene oxide paper is currently the focus of significant research efforts with a view to its practical use as components in, e.g., Li ion batteries, fuel cells, supercapacitors or electromechanical actuators [40-43]. In the present work, both global [e.g., Raman spectroscopy and X-ray photoelectron spectroscopy (XPS)] and local [scanning tunneling microscopy (STM)] characterization techniques have been employed to examine the graphitized films, revealing that atomic-sized (point) defects can be eliminated altogether from the basal planes of the reduced graphene oxide sheets at sufficiently high annealing temperatures. Significantly, such extensive healing easily affords the highest values of electrical 
conductivity that have so far been documented for films obtained from graphene oxide, approaching those typical of pristine, highly oriented graphites.

\section{Experimental}

The graphitization experiments were carried out on free-standing, paper-like films of chemically reduced graphene oxide, which were prepared as follows. First, graphite oxide was synthesized from natural graphite powder (Fluka 50870) by the Hummers method, using $\mathrm{H}_{2} \mathrm{SO}_{4}, \mathrm{NaNO}_{3}$ and $\mathrm{KMnO}_{4}$, as described elsewhere [44]. After being rinsed with $10 \% \mathrm{HCl}$ solution and thoroughly washed with deionized water, the oxidation product was suspended in deionized water, sonicated in an ultrasound bath cleaner (J.P. Selecta Ultrasons system, $40 \mathrm{kHz}$ ) for $4 \mathrm{~h}$ and centrifuged (Eppendorf 5424 microcentrifuge) at $10000 \mathrm{~g}$ for $10 \mathrm{~min}$. The resulting supernatant (top $~ 75 \%$ of the total volume), which is a dispersion of single-layer graphene oxide sheets, was collected for further use and its concentration determined by means of UV-vis absorption spectroscopy based on a previously reported procedure [45]. Aqueous graphene oxide dispersions $\left(0.1 \mathrm{mg} \mathrm{mL}^{-1}\right)$ were chemically reduced with hydrazine monohydrate $(0.05 \mu \mathrm{L}$ of reductant per $\mathrm{mL}$ of dispersion) by heating at $95{ }^{\circ} \mathrm{C}$ for $1 \mathrm{~h}$ under basic conditions ( $\mathrm{pH} \sim 10$, adjusted by adding 2 $\mu \mathrm{L}$ of $25 \%$ ammonia solution per $\mathrm{mL}$ of dispersion). This procedure has been formerly shown to afford stable dispersions of well-reduced graphene oxide sheets [46]. Finally, a given volume of the reduced dispersion (typically $250 \mathrm{~mL}$ ) was vacuum-filtered through a silver membrane filter $(47 \mathrm{~mm}$ in diameter and $0.2 \mu \mathrm{m}$ of pore size, from Whatman). After drying under ambient conditions and peeling off the membrane filter, a free-standing paper-like film of reduced graphene oxide, denoted as HG, was obtained.

Reduced graphene oxide paper samples were subjected to high temperature annealing in a graphite furnace under argon flow $\left(2 \mathrm{~L} \mathrm{~min}^{-1}\right)$. For reasons that will be commented upon below, a two-step heat treatment procedure was implemented: the paper-like films were first annealed at $1500{ }^{\circ} \mathrm{C}$, and then, in a second independent run, annealed again at a higher temperature (1800, 2100, 2400 or $2700{ }^{\circ} \mathrm{C}$ ). The following heating rates were applied: $50{ }^{\circ} \mathrm{C} \mathrm{min}^{-1}$ from room temperature to $700{ }^{\circ} \mathrm{C}, 100{ }^{\circ} \mathrm{C}$ $\mathrm{min}^{-1}$ from 700 to $1000{ }^{\circ} \mathrm{C}, 25^{\circ} \mathrm{C} \mathrm{min}{ }^{-1}$ between 1000 and $2000{ }^{\circ} \mathrm{C}$, and $10{ }^{\circ} \mathrm{C}$ min $^{-1}$ between 2000 and $2700{ }^{\circ} \mathrm{C}$. Once it was reached, the target temperature was kept constant for $1 \mathrm{~h}$ and finally the samples were cooled down to room temperature while maintaining the same argon flow. The graphitized samples were denoted as HG1500 (film heat treated only once, at $1500{ }^{\circ} \mathrm{C}$ ) and HG1500-T (film first heat treated at $1500{ }^{\circ} \mathrm{C}$ and then at a temperature $\mathrm{T}$; e.g., HG1500-1800, HG1500-2100, etc).

Characterization was carried out by means of UV-vis absorption spectroscopy (only for the starting aqueous dispersions), X-ray diffraction (XRD), Raman spectroscopy, thermogravimetric analysis (TGA), STM, atomic force microscopy (AFM), XPS and measurement of electrical conductivity of the films. UV-vis absorption spectra were recorded with a double-beam He $\lambda$ ios $\alpha$ spectrophotometer (Thermo Spectronic). XRD was performed on a Bruker D8 Advance diffractometer using $\mathrm{Cu} \mathrm{K} \alpha$ radiation $(\lambda=0.154 \mathrm{~nm})$ at a step size and time of $0.02^{\circ}(2 \theta)$ and $1 \mathrm{~s}$, respectively. Raman spectra were obtained with a Horiba Jobin-Yvon LabRam apparatus at a laser excitation wavelength of $532 \mathrm{~nm}$. To avoid damage to the samples, a low incident laser power $(\sim 1 \mathrm{~mW})$ was used. TGA was accomplished

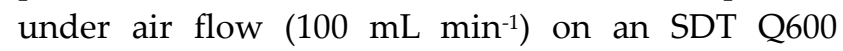
thermobalance (TA Instruments) at a heating rate of $10{ }^{\circ} \mathrm{C} \mathrm{min}^{-1}$, using Pt crucibles. AFM and STM measurements were performed with a Nanoscope IIIa Multimode apparatus (Veeco) under ambient conditions. AFM was carried out in the tapping mode of operation with rectangular silicon cantilevers. STM images were recorded using mechanically prepared $\mathrm{Pt} / \mathrm{Ir}$ (80/20) tips. A SPECS spectrometer, working with a monochromatic $\mathrm{Al} \mathrm{K \alpha}$ X-ray source $(100 \mathrm{~W})$ at a pressure of $10^{-7} \mathrm{~Pa}$, was used for the XPS measurements. The electrical conductivity of the different paper-like films, cut into $12 \times 12 \mathrm{~mm}^{2}$ squares, was determined by the van der Pauw method with a home-made setup (Agilent 6614C DC power supply and Fluke 45 digital multimeter). 

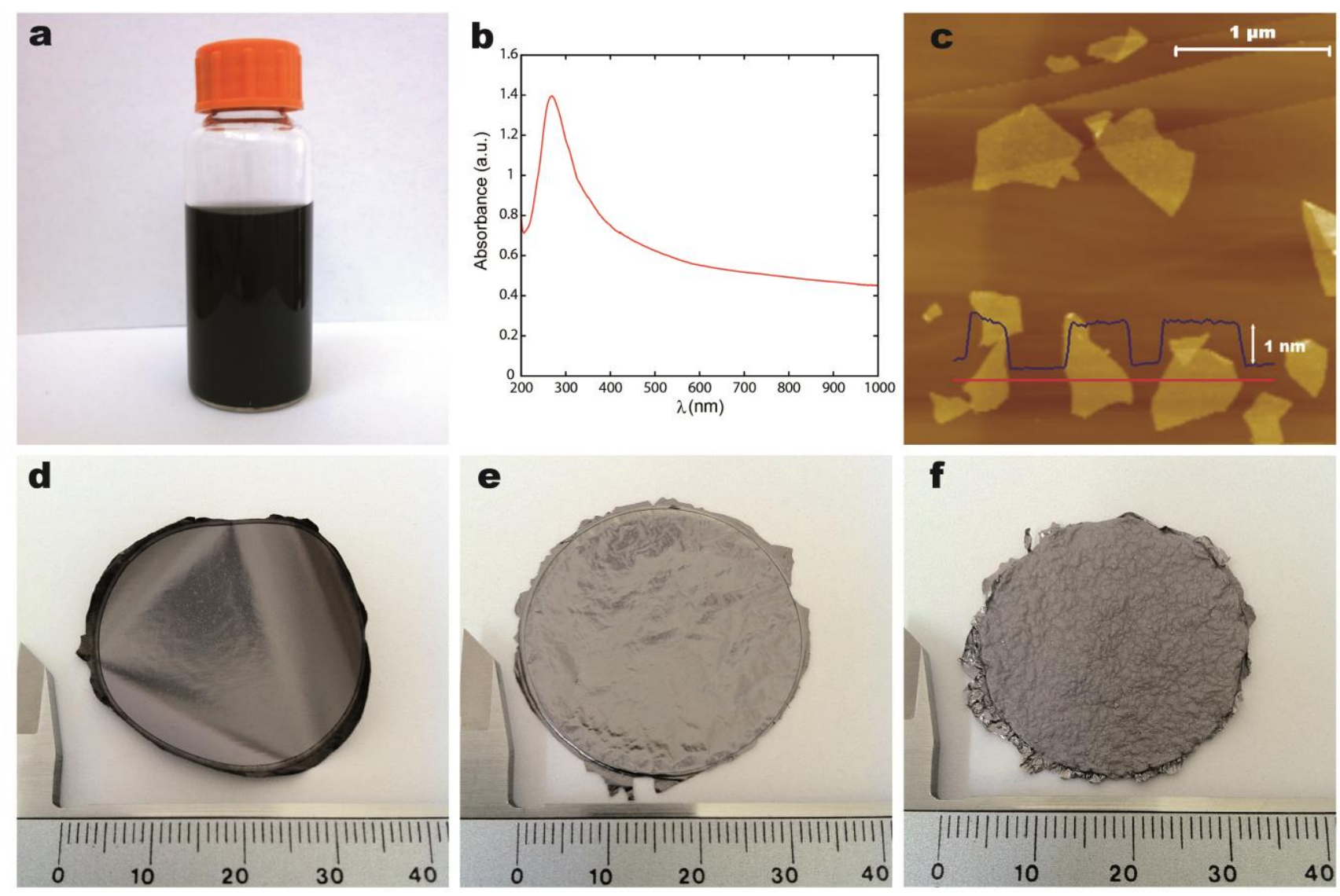

Figure 1 Digital photograph (a) and UV-vis absortion spectrum (b) of the starting aqueous dispersion of reduced graphene oxide. (c) Representative AFM image of the reduced graphene oxide sheets deposited onto HOPG. (d-f) Digital photographs of the starting HG film (d) as well as of annealed films HG1500 (e) and HG1500-2700 (f). The numbers in the scale in (d-f) correspond to millimeters.

\section{Results and discussion}

Fig. 1(a) shows a digital photograph of a vial containing a sample of the aqueous, hydrazine-reduced graphene oxide dispersion $(0.1$ $\mathrm{mg} \mathrm{mL}^{-1}$ ) that was used for the preparation of the paper-like films. In contrast to the yellow-brown color of its unreduced counterpart (not shown), such dispersion exhibits a deep black tone, suggesting that the graphene oxide sheets have been effectively reduced. This point can be corroborated by UV-vis absorption spectroscopy, which has proved to be a useful technique to monitor the reduction degree of graphene oxide [46]. The UV-vis absorption spectrum of the reduced suspensions prepared here (Fig. 1(b)) displays a peak at about $268 \mathrm{~nm}$ and strong absorbance in the whole wavelength range above $268 \mathrm{~nm}$, indicating that the hydrazine reduction step succeeded in deoxygenating the graphene oxide sheets to a significant extent [46,47]. Further prove of the effective reduction of graphene oxide by XPS analysis is given in Fig. S-1 in the Electronic Supplementary Material. A representative AFM image of the reduced sheets after being drop-cast from their aqueous suspension onto a highly oriented pyrolytic graphite (HOPG) substrate is shown in Fig. 1(c). The sheets display irregular shapes and are typically a few to several hundred nanometers in lateral size, with an apparent thickness, determined relative to the HOPG substrate, of $\sim 1 \mathrm{~nm}$ (see superimposed line profile in Fig. 1(c)). Such apparent thickness implies that the sheets are individual, single-layer objects (i.e., single-layer reduced graphene oxide) [45,47]. Vacuum-filtering this reduced suspension afforded free-standing papers with a shiny metallic appearance (Fig. 1(d)), which were used in the subsequent heat treatments.

It should be stressed that paper-like films of 
chemically reduced graphene oxide were deliberately chosen for the graphitization experiments as opposed to films of unreduced graphene oxide: during high temperature annealing, the oxygen functional groups present on both unreduced and chemically reduced graphene oxide are expected to be removed in the form of $\mathrm{CO}$ and $\mathrm{CO}_{2}$ molecules $[15,19,48-50]$. This implies that carbon atoms should be abstracted from the graphene lattice, and as a result, structural defects such as atomic vacancies can be created [49], which would subsequently have to be healed if a pristine graphitic lattice is to be restored. Because chemical reduction with hydrazine removes a large fraction of the oxygen originally present in graphene oxide without significant evolution of carbon atoms [51-53] and thus without creating vacancies or other defects, the amount of structural defects brought about in chemically reduced graphene oxide upon stripping its residual oxygen functional groups by high temperature annealing should be much smaller than that generated on its unreduced, oxygen-crowded counterpart. Bearing a lower density of heat treatment-induced defects in its carbon lattice, the subsequent healing of the chemically reduced sheets should be easier to achieve compared to that of unreduced graphene oxide. Furthermore, very recent work has shown that paper-like films of unreduced graphene oxide tend to crack up at annealing temperatures as low as $800{ }^{\circ} \mathrm{C}$ [36], whereas the structural integrity of papers made of chemically reduced graphene oxide studied here was preserved for all the annealing temperatures up to $2700{ }^{\circ} \mathrm{C}$ (Fig. 1(e) and (f)).

The previous reasoning also points to the idea that complete healing of reduced graphene oxide by means of heat treatment to yield a pristine graphitic lattice can be basically viewed as a two-step process, comprising first the removal of its oxygen functional groups and then the repair of the structural defects left behind in the carbon lattice by the evolving oxygen. Thus, we hypothesized that graphitization of the reduced graphene oxide paper could be similarly approached in two annealing steps: the first one would be carried out at a moderately high temperature to remove most of the residual oxygen

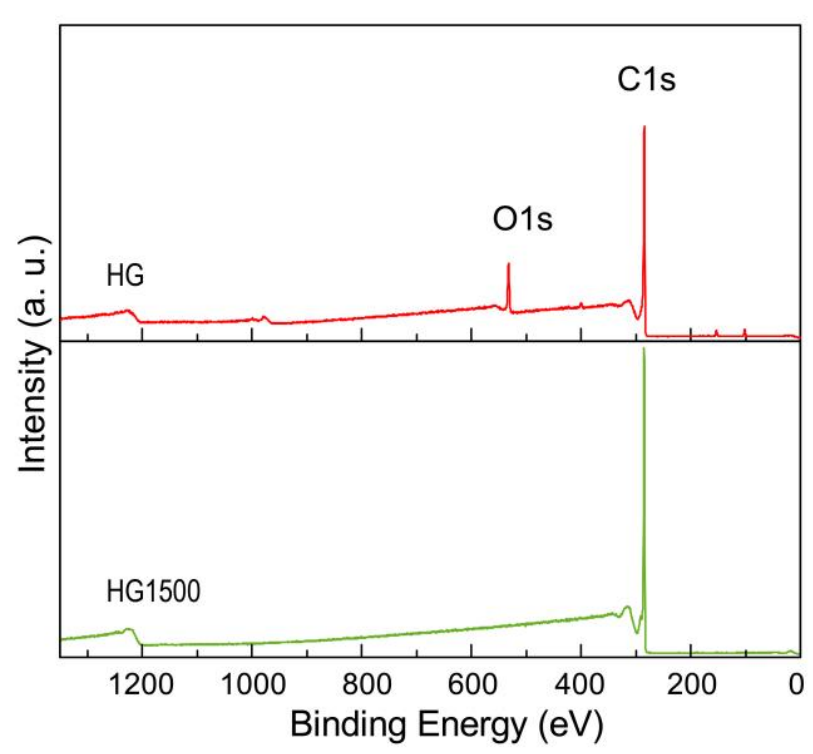

Figure 2 Survey X-ray photoelectron spectra for the starting HG film (red plot) and annealed film HG1500 (green).

that remains in reduced graphene oxide, while a second annealing at a higher temperature would allow the enduring lattice defects and structural imperfections to repair. In this way, the two steps could be studied independently. We chose the temperature of the first annealing step to be $1500{ }^{\circ} \mathrm{C}$ based on previous work which suggested that a significant fraction of the original oxygen groups of graphene oxide (i.e., mostly hydroxyls and epoxies) are converted to carbonyls and ethers upon heat treatment [49]. The latter functional groups are thermodynamically very stable and probably require temperatures in excess of $1000{ }^{\circ} \mathrm{C}$ to be removed $[49,54]$. In fact, we observed by means of XPS that practically all the oxygen present in the as-prepared, chemically reduced graphene oxide films could be eliminated during the first annealing step at $1500{ }^{\circ} \mathrm{C}$, up to the point where oxygen was below the usual detection level for this technique. This point can be appreciated from Fig. 2, where XPS survey spectra for the starting reduced graphene oxide film (sample $\mathrm{HG}$ ) and the film subjected to a single annealing at $1500{ }^{\circ} \mathrm{C}$ (sample HG1500) are presented. The O/C atomic ratios derived from the XPS data for these two and the remaining samples are given in Table 1. Such a ratio was 0.102 for the starting HG film (down from 0.430 for the unreduced graphene oxide film) but decreased to less than 0.001 for sample HG1500. As could be anticipated, the subsequent higher 
Table 1. O/C atomic ratio derived from XPS; interlayer spacing $\left(\mathrm{d}_{002}\right)$ and apparent crystallite size in the $c$ direction $\left(\mathrm{L}_{\mathrm{c}}\right)$ obtained by XRD; integrated intensity ratio of the $\mathrm{D}$ and $\mathrm{G}$ bands $\left(\mathrm{I}_{\mathrm{D}} / \mathrm{I}_{\mathrm{G}}\right)$ and apparent in-plane crystallite size $\left(\mathrm{L}_{\mathrm{a}}\right)$ calculated through the Tuinstra-Koenig (T-K) and Cançado equations from Raman spectra; and electrical conductivity of the starting reduced graphene oxide film, films annealed at different temperatures and HOPG.

\begin{tabular}{lccccccc}
\hline Sample & O/C at. ratio & $\mathrm{d}_{002}(\AA)$ & $\mathrm{L}_{\mathrm{C}}(\mathrm{nm})$ & $\mathrm{I}_{\mathrm{D}} / \mathrm{I}_{\mathrm{G}}$ & $\begin{array}{c}\mathrm{L}_{\mathrm{a}}(\mathrm{nm}) \\
(\mathrm{T}-\mathrm{K})\end{array}$ & $\begin{array}{c}\mathrm{L}_{\mathrm{a}}(\mathrm{nm}) \\
(\text { Cançado })\end{array}$ & $\begin{array}{c}\text { Conductivity } \\
\left(\mathrm{S} \mathrm{m}^{-1}\right)\end{array}$ \\
\hline HG & 0.102 & 3.944 & 1.2 & 1.83 & 2.4 & 10.5 & 7,962 \\
HG1500 & $<0.001$ & 3.416 & 7.2 & 0.72 & 6.1 & 26.7 & $2.18 \times 10^{5}$ \\
HG1500-1800 & $<0.001$ & 3.431 & 10.2 & 0.11 & 40.6 & 177.2 & $1.71 \times 10^{5}$ \\
HG1500-2100 & $<0.001$ & 3.423 & 13.6 & 0.09 & 50.6 & 221.2 & $2.31 \times 10^{5}$ \\
HG1500-2400 & $<0.001$ & 3.378 & 27.4 & 0.07 & 62.5 & 273.1 & $3.34 \times 10^{5}$ \\
HG1500-2700 & $<0.001$ & 3.366 & 35.6 & 0.02 & 236.6 & $1,033.6$ & $5.77 \times 10^{5}$ \\
HOPG & $<0.001$ & 3.355 & 52.8 & 0 & -- & -- & $1.69 \times 10^{6}$ \\
\hline
\end{tabular}

temperature treatments (second annealing step) also yielded negligible amounts of oxygen (spectra not shown). These results demonstrate that under adequate conditions a virtually complete elimination of oxygen groups from reduced graphene oxide is possible. In contrast, residual oxygen was detected in all previous attempts to restore the original graphene lattice reported in the literature, even when temperatures above $1500{ }^{\circ} \mathrm{C}$ were used [39]. Additional information on the XPS results of the films under study can be found in Fig S-2 in the Electronic Supplementary Material.

The global structural evolution of the heat-treated paper-like samples was investigated by means of XRD and Raman spectroscopy. The X-ray diffractograms of the different films and of highly oriented pyrolytic graphite (HOPG) as a reference sample are presented in Fig. 3(a). The non-annealed film (sample HG) displayed a relatively weak and broad diffraction peak at $\sim 22.5^{\circ}(2 \theta)[40,55]$, which corresponds to an interlayer spacing, d002, of $3.944 \AA$ (see Table 1). After the first annealing step (sample HG1500) the diffraction peak became much sharper and the do02 spacing decreased to $3.416 \AA$. A further decrease was observed, down to $3.366 \AA$ and thus approaching the HOPG value (3.355 $\AA$ ), following the second annealing, but only for the highest heat treatment temperatures $\left(2400\right.$ and $2700{ }^{\circ} \mathrm{C}$; see Table 1). By contrast, the do02 spacing for the samples annealed at 1800 and $2100{ }^{\circ} \mathrm{C}$ (3.431 and $3.423 \AA$, respectively) was slightly larger than that measured for sample HG1500. A possible explanation to account for this a priori unexpected result will be given below. The decreasing width of the diffraction peak was reflected in the evolution of the apparent crystallite size in the $c$ direction, i.e. the $\mathrm{L}_{c}$ parameter (Table 1), which was as low as $1.2 \mathrm{~nm}$ for the starting HG film, increased to $7.2 \mathrm{~nm}$ following the first heat treatment and finally reached $35.6 \mathrm{~nm}$ after the second annealing at $2700{ }^{\circ} \mathrm{C}$. Again, this value was relatively close, but not identical, to that of the HOPG reference sample $(52.8 \mathrm{~nm})$.

Fig. 3(b) shows first- and second-order Raman spectra of the investigated samples. The first order spectrum $\left(1100-1700 \mathrm{~cm}^{-1}\right)$ is dominated by three features: a strong peak at $\sim 1583 \mathrm{~cm}^{-1}$ (G band) and two additional peaks at 1355 and $1625 \mathrm{~cm}^{-1}$, which correspond to the defect-related $\mathrm{D}$ and $\mathrm{D}^{\prime}$ bands, respectively $[56,57]$. The Raman spectrum of the non-annealed HG film exhibited strong D and $\mathrm{D}^{\prime}$ peaks (relative to the $G$ band), denoting a large amount of structural defects in its carbon lattice 

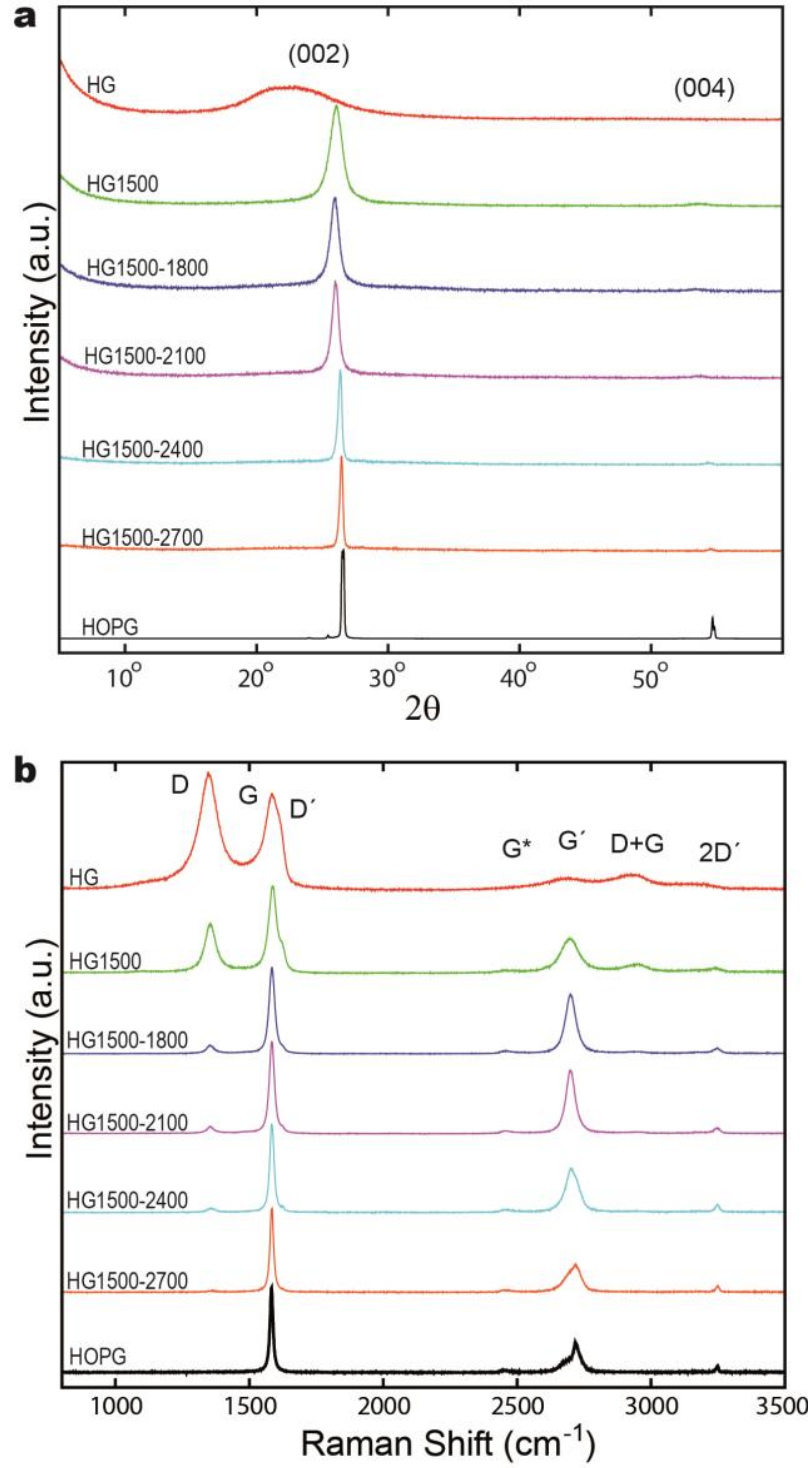

Figure 3 XRD patterns (a) and Raman spectra (b) for the starting HG film (red plots), heat-treated films HG1500 (green), HG1500-1800 (dark blue), HG1500-2100 (purple), HG1500-2400 (light blue) and HG1500-2700 (orange), and HOPG (black).

$[45,51]$. Following the first heat treatment at $1500{ }^{\circ} \mathrm{C}$, the intensity of both defect-related bands decreased markedly, but in any case they were still relatively large, which suggests that a significant amount of disorder remained in the structure. At least in relative terms, the healing of defects appeared to be much more extensive during the second heat treatment step, as noticed by the evolution of the integrated intensity ratio of the $\mathrm{D}$ and $\mathrm{G}$ bands $\left(\mathrm{I}_{\mathrm{D}} / \mathrm{IG}_{\mathrm{G}}\right)$ for the different samples (Table 1), which provides a quantitative measure of the amount of defects present in graphitic materials [56,57]. The ID/IG ratio was determined to be $1.83,0.72$ and 0.02 for samples HG, HG1500 and HG1500-2700, respectively. Such observation, together with the XPS results, lends support to the above assumption that a thorough healing of reduced graphene oxide by high temperature annealing proceeds mainly by first removing its residual oxygen groups and then repairing the structural defects left behind in the carbon lattice by the oxygen groups. The ID/IG ratio can be used to derive an apparent in-plane crystallite size ( $\mathrm{La}$ parameter), either by means of the well-known Tuinstra-Koenig equation [ 58 ] or, alternatively, based on the formula proposed more recently by Cançado et al [59]. La values obtained from both equations were somewhat different (Table 1 ), and were up to $\sim 10 \mathrm{~nm}$ for the starting HG film, as is typical for chemically reduced graphene oxide [22], but reached values in the range of several hundred nanometers for sample HG1500-2700. The meaning of this parameter for the highly graphitized films will be discussed below in the light of the STM results.

The second-order Raman spectra (i.e., 2300-3300 $\mathrm{cm}^{-1}$ region in Fig. 3(b)) display up to four different bands $\left(G^{*}, G^{\prime}, D+G\right.$ and $\left.2 D^{\prime}\right)$, that are also characteristic of graphitic materials $[57,60]$. The most relevant one is the $G^{\prime}$ peak, located at about 2695 $\mathrm{cm}^{-1}$, which is the overtone of the $\mathrm{D}$ band (for this reason, it is usually referred to as the $2 \mathrm{D}$ band). Its intensity is sensitive to the presence of structural disorder (i.e., it tends to decrease in the presence of large amounts of disorder; e.g., sample HG), but most significantly, analysis of its lineshape reveals information about the three-dimensional stacking order of the sample [60]. As shown in detail in Fig. 4, the $G^{\prime}$ band for a graphitic material with ordered stacking (AB Bernal stacking) such as HOPG exhibits a highly asymmetric lineshape that can be fitted with two Lorentzian components ( $\mathrm{G}^{\prime} 3 \mathrm{DA}$ and $\left.\mathrm{G}^{\prime} 3 \mathrm{DB}\right)$. By contrast, a material in which the graphene layers are randomly rotated with respect to each other around the $c$ axis (turbostratic stacking) possesses a $G^{\prime}$ band that is completely symmetric and can thus be fitted with a single Lorentzian component $\left(\mathrm{G}^{\prime} \mathrm{D}\right)$, located in-between the $G^{\prime}{ }^{\prime}{ }^{2} A$ and $G^{\prime}{ }_{3 D B}$ lines. In the present case, because the reduced graphene oxide films were produced by vacuum-assisted assembly of the 


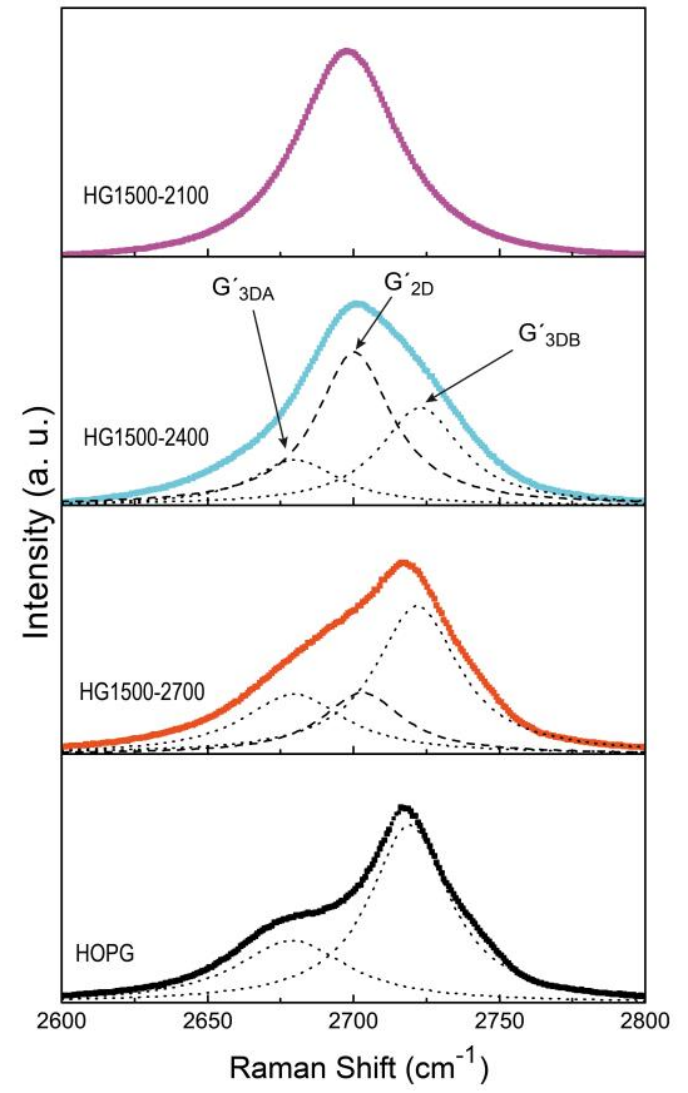

Figure $4 \mathrm{G}^{\prime}$ Raman band spectra for the heat-treated films HG1500-2100 (purple plot), HG1500-2400 (light blue) and HG1500-2700 (orange), as well as for HOPG (black).

individual, single-layer sheets from their aqueous dispersion, the sheets are expected to be randomly piled over each other, leading to turbostratic stacking. In fact, the $G^{\prime}$ band displayed an altogether symmetric lineshape for films heat-treated up to $2100{ }^{\circ} \mathrm{C}$ in the second annealing step (see Fig. 3(b) and 4). However, asymmetry gradually arose at higher annealing temperatures, suggesting that $\mathrm{AB}$ Bernal stacking was developed to some degree. To evaluate the extent of three-dimensional ordering achieved, it was assumed that they consisted of a mixture of regions with turbostratic stacking and regions with $A B$ Bernal stacking. Consequently, their $\mathrm{G}^{\prime}$ band was fitted using a combination of the $\mathrm{G}_{2 \mathrm{D}}$, $\mathrm{G}^{\prime}$ 3DA and $\mathrm{G}^{\prime}$ зов Lorentzian components (Fig. 4), so that the fraction, $R$, of sample exhibiting Bernal stacking was given by [60]

$$
R=\frac{I_{G_{3 D B}^{\prime}}}{I_{G_{3 D B}^{\prime}}+I_{G_{2 D}^{\prime}}}
$$

Using Eq. (1), the fraction of Bernal-stacked regions

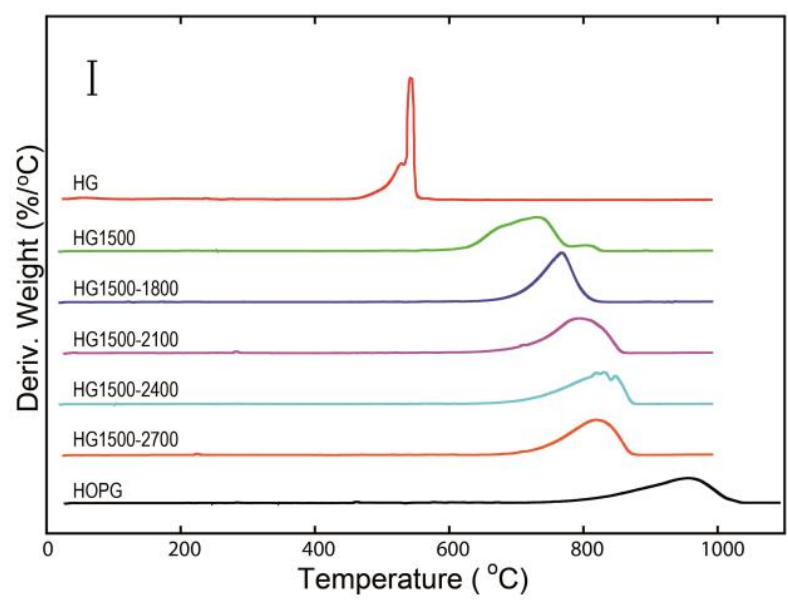

Figure 5 Differential thermogravimetric plots for the starting $\mathrm{HG}$ film (red plot), heat-treated films HG1500 (green), HG1500-1800 (dark blue), HG1500-2100 (purple), HG1500-2400 (light blue) and HG1500-2700 (orange), as well as for HOPG (black). The vertical scale in the upper left part of the graph corresponds to a weight derivative value of $1 \% /{ }^{\circ} \mathrm{C}$.

was determined to be negligible for films heat-treated at $2100{ }^{\circ} \mathrm{C}$ and below, $\sim 40 \%$ for sample HG1500-2400 and $\sim 70 \%$ for sample HG1500-2700, indicating that a significant degree of three-dimensional ordering can be attained at the highest annealing temperature. In other words, the films annealed at the highest temperatures are approaching to highly oriented graphites.

The reactivity of graphitic materials, and in particular their reactivity towards oxidation, is known to be strongly dependent on the amount and type of defects present in their structure [61-64]. To assess the effect of annealing on the film reactivity, they were subjected to TGA under flowing air. The thermogravimetric (TG) plots were dominated by a sharp mass loss step that can be ascribed to gasification of the material by reaction with oxygen. Fig. 5 shows the first derivative of the TG plots, i.e. the differential thermogravimetric (DTG) plots, for the paper-like films and HOPG. The peak in the DTG plots indicates the temperature at which gasification of the sample was fastest. For the non-annealed HG film, gasification took place at a relatively low temperature $\left(\sim 540{ }^{\circ} \mathrm{C}\right)$, implying a high reactivity that can be attributed to the distorted carbon structure induced by the large amount of residual oxygen groups that this film contains. The first annealing $\left(1500{ }^{\circ} \mathrm{C}\right)$ brought about a remarkable up-shift in the gasification temperature to $\sim 731{ }^{\circ} \mathrm{C}$ 
and therefore a significantly less reactive material. The second annealing step gave rise to more modest changes (e.g., gasification at $\sim 768{ }^{\circ} \mathrm{C}$ for HG1500-1800), and such changes were increasingly marginal with increasing heat treatment temperature. Considering that a relatively large amount of defects is eliminated in this second stage (see Raman results, Fig. 3(b)), such observation suggests that the defects remaining after annealing at $1500{ }^{\circ} \mathrm{C}$ are associated to a lower reactivity compared to that of the original defects in the reduced graphene oxide film. The reactivity of films annealed at the highest temperature (HG1500-2700) still differed considerably from that of HOPG (gasification at $\sim 956^{\circ} \mathrm{C}$ ), implying a somewhat less pristine structure for the former, in agreement with the XRD and Raman spectroscopy data.

The electrical conductivity is also a highly sensitive indicator of the presence of defects in graphitic carbon. Defects generally act as scattering sites for the charge carriers, thus hampering their mobility and in turn decreasing the overall conductivity of the material [64]. The values measured for the paper-like films and HOPG are given in Table 1. The non-annealed HG film exhibited an electrical conductivity $\left(7,960 \mathrm{~S} \mathrm{~m}^{-1}\right)$ that is comparable to the values previously reported for chemically reduced graphene oxide paper produced by similar means $[46,47,55]$. A very large increase in conductivity (up to $218,000 \mathrm{~S} \mathrm{~m}^{-1}$ ) was observed following the first annealing step, whereas further improvements could be attained with the second annealing, but only at the highest temperatures (2400 and $2700{ }^{\circ} \mathrm{C}$ ), reaching a maximum value of $577,000 \mathrm{~S}$ $\mathrm{m}^{-1}$ for sample HG1500-2700. To the best of our knowledge, this is by far the largest value of electrical conductivity that has been documented for a material derived from graphene oxide. The former highest figure was very recently achieved for a chemically reduced graphene oxide paper that had been heat-treated at $1900{ }^{\circ} \mathrm{C}\left(160,000 \mathrm{~S} \mathrm{~m}^{-1}\right)$ [39]. For comparison, a conductivity as high as $1,690,000 \mathrm{~S} \mathrm{~m}^{-1}$ was estimated here for the HOPG sample, which is consistent with the values reported in the literature and close to those measured for single-crystal graphite $\left(\sim 2,500,000 \mathrm{~S} \mathrm{~m}^{-1}\right)$ [65-67].

As previously hypothesized, with a two-step approach to the restoration of the originally pristine carbon lattice, it has been possible to remove all the oxygen in the reduced graphene oxide films at a relatively low annealing temperature and then, on films with no residual oxygen, to independently investigate the healing of defects at higher annealing temperatures. The fact that these two processes (removal of oxygen and healing of defects) take place at so different temperatures suggests that their activation energies differ significantly. Although, in a practical case, a single annealing at the highest temperatures (e. g., $2400{ }^{\circ} \mathrm{C}$ or $2700{ }^{\circ} \mathrm{C}$ ) could be used to completely restore the carbon lattice (see Fig. S-3 in the Electronic Supplementary Material), even in such a case, the process with the lowest activation energy would be much more probable and therefore it would occur much faster. Summing up, in practice the treatment can be done in one stage but anyhow the physical process of annealing would always take place in two steps.

The previous results have revealed the general structural and chemical evolution of reduced graphene oxide films upon graphitization treatments. It has been determined that the structural characteristics and other features (reactivity and electrical conductivity) of the highly graphitized films approach very significantly but do not match those of a model graphitic material such as HOPG. It is reasonable to argue that the key to understand this result lies in the evolution of the nanometer- and atomic-scale structure of the films during graphitization. Therefore, to shed light on this question the samples were examined by STM, a microscopy technique with enough resolution to image even atomic-sized (point) defects on graphitic materials [68-70]. Fig. 6 shows representative STM images of the nanometer-scale morphology for the as-prepared and different heat-treated films, with the overlaid line profiles indicating the typical height variations that were observed in each case. For the starting HG film (Fig. 6(a)), a relatively rough topography can be noticed, which is made up of more or less smooth features $\sim 10-30 \mathrm{~nm}$ in lateral size (with the exception of some elongated features; e.g., lower right part of Fig. 6(a)). Such a characteristic feature size is at least one order of magnitude smaller than the size of the reduced graphene oxide sheets that served as building blocks for the film (Fig. 1(c)). The origin of this topography 

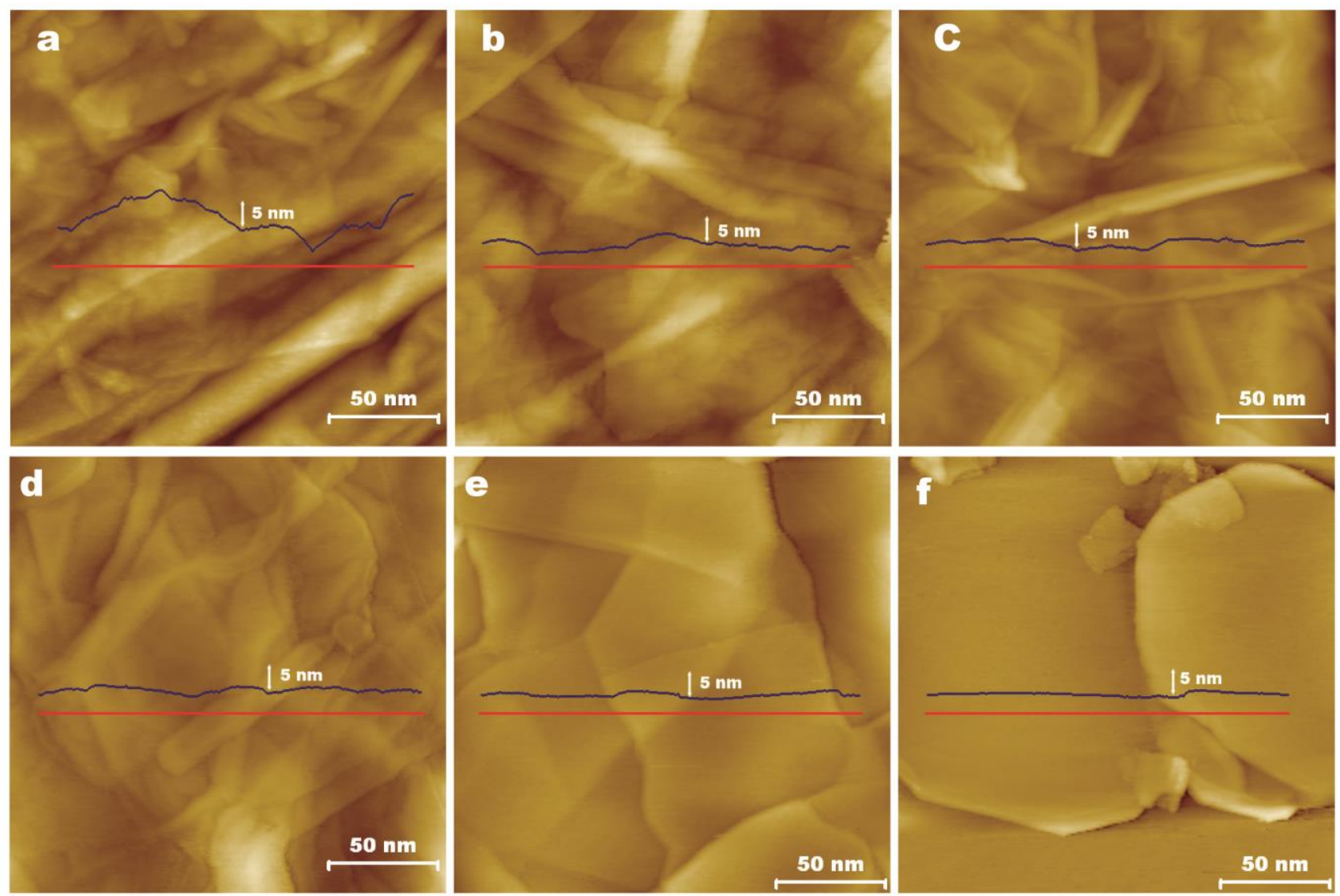

Figure 6 Nanometer-scale STM images for the starting HG film (a) and heat-treated films HG1500 (b), HG1500-1800 (c), HG1500-2100 (d), HG1500-2400 (e) and HG1500-2700 (f). Typical tunneling parameters were 100-300 pA (tunneling current) and $1500 \mathrm{mV}$ (bias voltage). In each case, a representative line profile (blue plot) taken along marked red line is shown overlaid onto the image.

can be mainly attributed to the random overlapping of the sheets while they are being stacked over each other during film formation. Although an isolated sheet on a flat substrate displays an essentially flat profile (Fig. 1(c)), the successive build-up of further sheets inevitably leads to their overlapping, thus giving rise to an irregular topography with lateral feature sizes that necessarily have to be smaller than those of the sheets. Following the first annealing (sample HG1500, Fig. 6(b)), the distinctive nanometer-scale morphology of the as-prepared film was essentially preserved. However, significant changes took place during the second heat-treatment step. Even at the lowest temperatures of 1800 and $2100{ }^{\circ} \mathrm{C}$ (Fig. 6(c) and (d), respectively), a clear trend towards a flat topography and features with larger lateral dimensions could be noticed, finally evolving into atomically flat and relatively large (a few hundred $\mathrm{nm}$ ) terraces at the highest annealing temperatures (2400 and $2700{ }^{\circ} \mathrm{C}$, Fig. 6(e) and (f), respectively). Such a progression is basically consistent with the emergence of a high degree of graphitic order in the films (as already revealed by XRD and Raman spectroscopy), the large terraces being ascribed to well-developed basal planes.

The structural evolution of the films upon graphitization was also investigated by STM on the atomic scale. Some representative images are shown in Fig. 7. In agreement with previous STM studies of individual, chemically reduced graphene oxide sheets [45], the as-prepared HG film did not exhibit long-range order on the atomic scale (Fig. 7(a)). It is well-known that highly ordered graphitic surfaces display a perfect triangular pattern (or, in some cases, a honeycomb pattern) with a periodicity of $\sim 0.25 \mathrm{~nm}$ in their STM images [71]. By contrast, although atomic-sized features were routinely observed for sample HG, such features were organized into very 

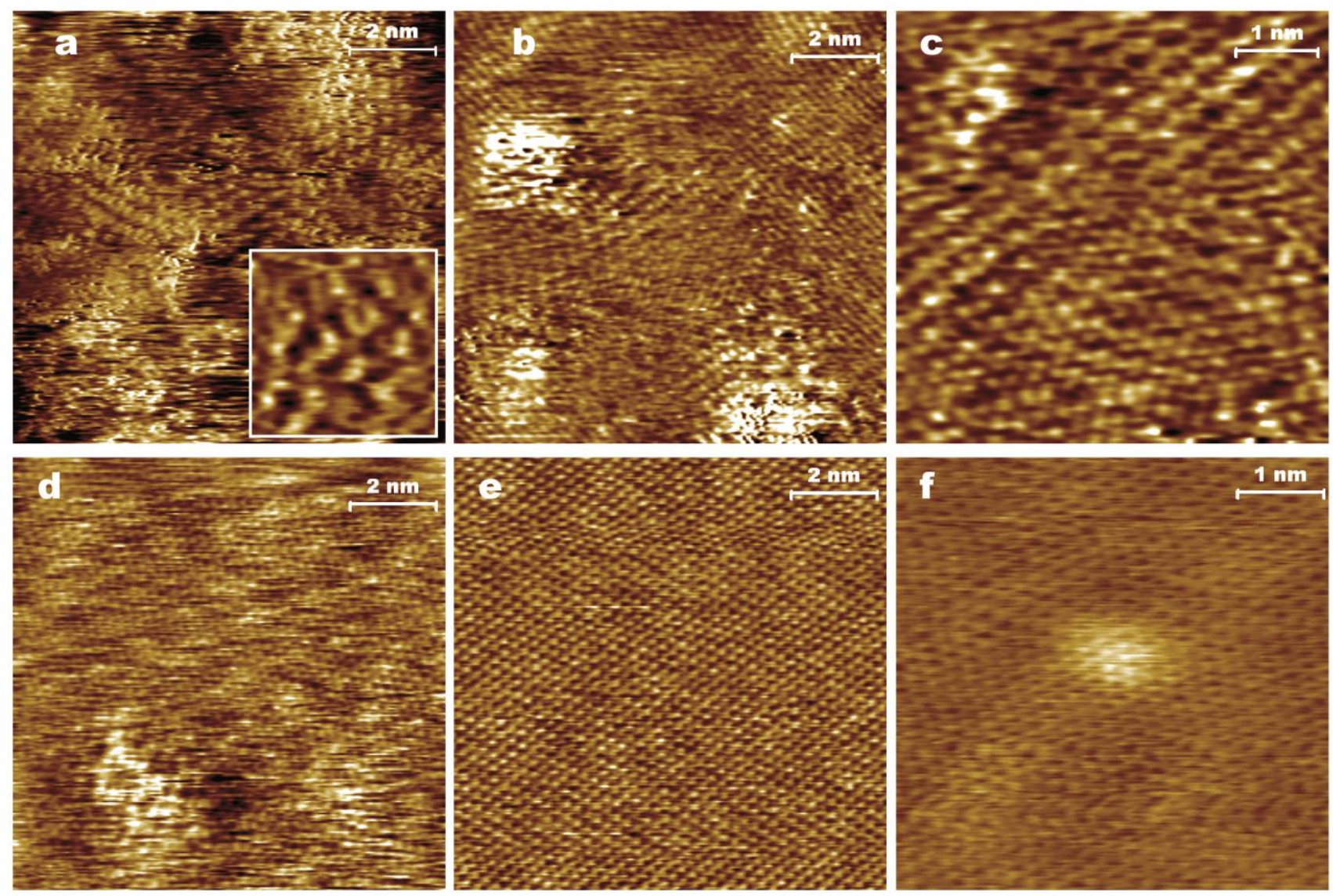

Figure 7 Atomic-scale STM images for the starting HG film (a) and heat-treated films HG1500 (b,c), HG1500-1800 (d) and HG1500-2700 (e,f). Typical tunneling parameters: 1-2 nA (tunneling current) and 5-100 mV (bias voltage). The inset to (a) is a detailed $2 \times 2 \mathrm{~nm}^{2}$ image showing the highly distorted atomic-scale patterns of the HG film.

small $(\sim 3-4 \mathrm{~nm})$ domains that usually lacked themselves an ordered triangular (or honeycomb) structure. This point can be better appreciated in the detailed images of a given domain (e.g., inset to Fig. $7(\mathrm{a}))$. The disorderly arrangement of atomic-sized features can be associated to the presence of different types of defects in the carbon structure. Such defects should include the relatively abundant number of oxygen-containing groups that have been measured on the HG film (see XPS results above), but also probably non-hexagonal carbon rings (e.g., 5or-7-membered rings), which have been suggested to form in reduced graphene oxide $[20,21]$. The strain induced by these defects on the carbon lattice can be expected to alter significantly its local electronic structure (compared to that of the perfect graphitic lattice), which is what the STM actually probes [72]. Furthermore, because the defects are most probably randomly arranged even on a local scale $[20,21]$, the resulting atomic-scale STM images should also display disorganized patterns, as actually observed (inset to Fig. 7(a)).

While the first annealing step did not bring about very significant changes to the nanometer-scale morphology of the films (compare Fig. 6(a) and (b)), their atomic-scale structure did in fact experience a remarkable transformation. As shown in Fig. 7(b), the atomic-scale STM images of sample HG1500 were characterized by the presence of a large number of apparent protrusions (bright features) $\sim 2-3 \mathrm{~nm}$ wide scattered on an otherwise relatively ordered structure. The regions between the protrusions displayed in many cases the familiar triangular or honeycomb patterns archetypal of well-ordered graphitic systems, whereas in other instances these patterns were seen to be modulated by local superstructures (e.g., Fig. 7(c)). Such superstructures are known to reflect a modulation of electronic density arising in highly graphitic regions that lie in close proximity to defects [73]. The 
protrusions themselves generally exhibited locally distorted or unclear atomic patterns, and can be interpreted as isolated defects remaining in the film after its graphitic structure is largely restored as a result of the removal of its oxygen functionalities. On the atomic scale, the most obvious change taking place during the second annealing was the progressive disappearance of these isolated defects, as exemplified in Fig. 7(d) and (e) for samples HG1500-1800 and HG1500-2700, respectively. For the latter film, the large majority of the recorded $10 \times 10$ $\mathrm{nm}^{2}$ images were completely free from any defects and they always displayed long-range graphitic order (e.g., Fig. 7(e)). However, rather than protrusions with distorted atomic-scale pattern, the residual defects in this film were mostly smooth, dome-like bumps where the pristine graphitic structure was not altered (Fig. 7(f)), suggesting that they were of a different nature to those prevalent at the lowest annealing temperatures (sample HG1500, Fig, 7(b)). The dome-like features were from $\sim 1$ up to several nanometers in diameter and can in principle be attributed to interstitial species (e.g., clusters of carbon atoms) trapped in-between the topmost graphene layers [69].

As already noted above, the removal of the oxygen functionalities as $\mathrm{CO}$ and $\mathrm{CO}_{2}$ molecules from the HG film with heat treatment at $1500{ }^{\circ} \mathrm{C}$ should inevitably lead to the generation of a significant number of atomic vacancies/small holes on the carbon lattice [48-50]. It is also well established that atomic vacancies in graphitic structures induce an enhancement in the local density of electronic states near the Fermi level, so that the vacancy is imaged by STM as a protrusion (bright feature) with lateral dimensions (up to several nanometers) mainly determined by the number of missing carbon atoms [68,70]. The protrusions observed for sample HG1500 (Fig. 7(b)) are consistent with their being atomic vacancies and are therefore ascribed to such a type of defect generated in the sheets by the first annealing step. Now, at sufficiently high temperatures the atomic vacancies can be expected to become mobile and annihilate at, e.g., the sheet edges, leading to their effective removal $[70,74]$. Although a single-atom vacancy is thought to possess a significant mobility even at close to ambient temperature due to a relatively low migration barrier, the mobility of multi-atom vacancies should be extremely low, therefore requiring very high temperatures to annihilate. For instance, the migration barrier for a two-atom vacancy in graphene has been estimated to be $6-7 \mathrm{eV}$, compared to $\sim 1.2-1.4 \mathrm{eV}$ in the case of a single-atom vacancy [64]. The latter is indeed easily removed at temperatures of just a few hundred degrees Celsius [70]. Furthermore, the lateral size of the protrusions seen by STM for the HG1500 film (Fig. 7(b)) suggests that they mostly correspond to multi-atom vacancies and not to single-atom vacancies, which are associated to noticeably smaller protrusions $(\sim 0.6-0.9 \mathrm{~nm})$ [70]. Consequently, these atomic vacancies should be expected to disappear only at very high temperatures, as was indeed the case during the second annealing.

Based on the previous results, it can be concluded that the central sequence of events in the graphitization process of the reduced graphene oxide films is the following: (i) removal of residual oxygen groups/vacancy generation (first annealing step), and (ii) vacancy annihilation (second annealing step). This mechanism can explain the development of essentially pristine, defect-free graphitic basal surfaces at the highest heat treatment temperature (i.e., sample HG1500-2700, Fig. 7(e)). Nevertheless, such a process alone cannot account for the nanometer-scale evolution of the film morphology that was described in Fig. 6. The generation and annihilation of atomic vacancies within the reduced graphene oxide sheets is not expected to significantly alter the random stacking/overlapping configuration of the sheets in the film. Consequently, if the rough morphology of the starting HG film (Fig. 6(a)) is due to the random arrangement of the sheets and only vacancy generation/annihilation processes come into play, then such a morphology should be essentially preserved throughout the whole annealing process, which was obviously not the case (Fig. 6(f)). Because topography of atomically flat terraces was seen to develop instead, we have to conclude that most of the overlaps between neighboring sheets in the starting HG film were eliminated, mainly during the second annealing step. We propose that adjacent sheets in the film coalesce at the points where a given sheet overlaps with the edge of another sheet, so that the film eventually transforms into a stack of 

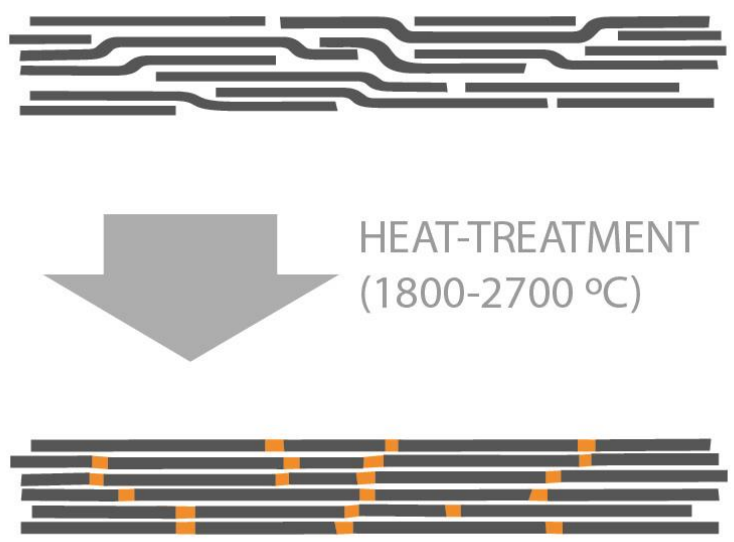

Figure 8 Schematic illustration of the sheet coalescence process in the reduced graphene oxide film during the second annealing step (1800-2700 $\left.{ }^{\circ} \mathrm{C}\right)$. The yellow-brown squares shown in the annealed film represent grain boundaries between neighboring domains in the resulting extended polycrystalline layers. non-overlapped, extended and flat graphitic layers. A schematic illustration of such structural transformation is given in Fig. 8. This interpretation is supported by very recent experiments carried out by Barreiro et al, who observed the coalescence of two overlapping graphene layers into one continuous sheet under Joule heating (temperatures up to $2000{ }^{\circ} \mathrm{C}$ ) inside a transmission electron microscope [75]. Even though the details of this transformation are currently not known and need to be elucidated, it can be argued that its main driving force is the elimination of the energy penalty associated to (i) the presence of a large amount of free graphene edges with dangling bonds and (ii) also probably the local strain of the carbon lattice due to curvature at the point where a sheet overlaps with the edge of another sheet in the starting film (Fig. 8).
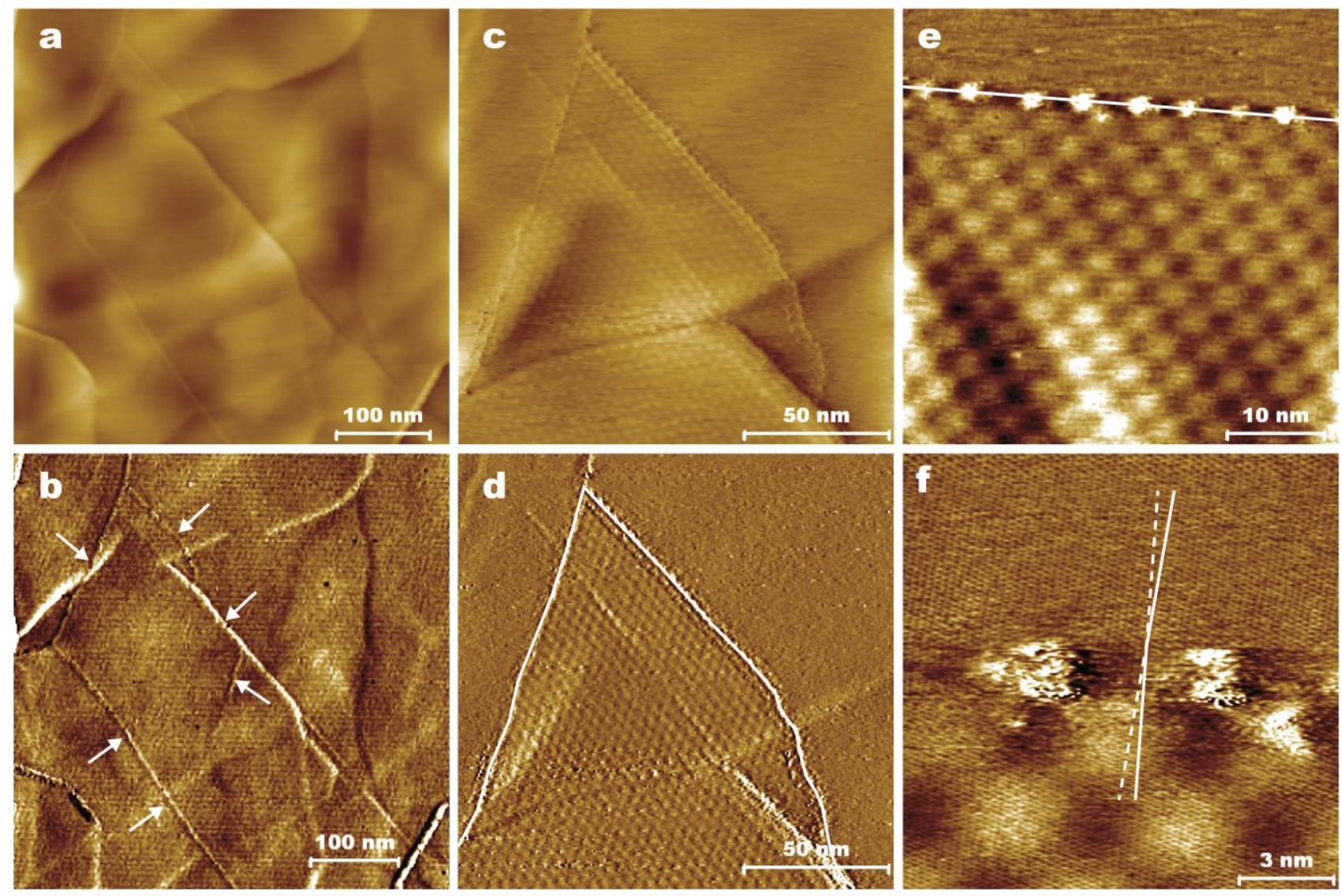

Figure 9 Height $(a, c)$ and corresponding current $(b, d)$ STM images of the HG1500-2700 film revealing the presence of grain boundaries [indicated by white arrows in (b) and by white lines in (d)]. (e,f) Height STM images of a grain boundary [indicated by a white line in (e)] and a Moiré pattern on one side of the boundary. The orientation of the carbon lattice on each side of the boundary is indicated by solid white lines in (f). Tunneling parameters: 0.2-0.8 nA (tunneling current) and $100-800 \mathrm{mV}$ (bias voltage). 
If the hypothesis of sheet coalescence is correct, and because the coalescing sheets should in principle exhibit random in-plane crystallographic orientations, then we would expect the resulting layers to be highly polycrystalline and thus contain a significant amount of grain boundaries. As a matter of fact, grain boundaries were ubiquitously seen by STM for sample HG1500-2700. In its low energy configuration, this type of line defect is thought to be made up of a string of alternating pentagons and heptagons [76]. This lattice distortion induces localization of electronic states near the Fermi level at the grain boundary, which is therefore detected by STM as an apparently elevated line $[71,77]$. Some examples of grain boundaries are shown in Fig. 9. Although this type of defect usually passed unnoticed among other features (e.g., surface and subsurface steps) in the low-resolution STM height images (Fig. 9(a)), it was readily detectable in their simultaneously recorded current images (Fig. 9(b); the grain boundaries are indicated by white arrows). The upper left part of Fig. 9(a) and b is presented in more detail in Fig. 9(c) and (d), respectively. The domain encircled inside the grain boundary as indicated by the white line in Fig. 9(d) exhibits a superperiodic structure (i.e., a Moiré pattern). Moiré patterns in graphitic systems result from the relative rotation of the two topmost graphene layers [71], and in the present work were frequently observed for the highly graphitized films. This is most probably a consequence of the random stacking of the reduced graphene oxide sheets in the starting HG film, which cannot be fully reverted to a perfect $A B$ stacking even at the highest annealing temperature. High resolution images of a grain boundary and associated Moiré pattern are given in Fig. 9(e) and (f). The grain boundary is indicated by a red line in Fig. 9(e), which is seen in this case as a string of discrete bright features. The carbon lattice is resolved in Fig. 9(f), revealing a slightly different $\left(\sim 3^{\circ}\right)$ crystallographic orientation on both sides of the boundary (see full and dotted white lines as a guide to the eye), which in turn confirms the nature of these line defects as grain boundaries and the development of continuous but polycrystalline layers upon graphitization. From Fig. 9(a,b) and additional STM images, the typical size of the grains was estimated to be a few hundred nanometers.
Hence, in the light of all the experimental evidence presented here, the graphitization process of the reduced graphene oxide films can be finally rationalized according to the following scheme: (i) First annealing step: removal of oxygen functional groups and generation of atomic vacancies within the reduced graphene oxide sheets; (ii) Second annealing step: annihilation of atomic vacancies and coalescence of overlapping sheets to yield continuous, polycrystalline layers that are essentially free of defects except for the presence of grain boundaries. Lastly, we note that such structural evolution can account for many of the results obtained by the different characterization techniques: (1) A highly graphitized film composed of polycrystalline layers having domains a few hundred nanometers large (i.e, sample HG1500-2700) can be expected to exhibit a non-negligible Raman D band arising from the grain boundaries, as was actually observed (see Fig. 3(b) and Table 1). (2) As the polycrystalline layers of sample HG1500-2700 were for the most part free of other defects, then its in-plane crystallite size (La) derived from Raman spectroscopy (Table 1) should be comparable to the size of the domains as observed by STM, which was the case as well. (3) In such a polycrystalline film, we cannot expect to have perfect AB Bernal stacking of the layers throughout the whole sample, as evidenced by the Moiré patterns of Fig. 9. This is in agreement with the analysis of the Raman $\mathrm{G}^{\prime}$ band of sample HG1500-2700 (Fig. 4), which revealed a significant fraction of non-Bernal stacking. (4) Many properties of the highly graphitized films can be expected to be dominated by the presence of a high density of grain boundaries (i.e., relatively small domain sizes). Specifically, in comparing the electrical conductivity and reactivity towards gasification of the HG1500-2700 film with the results obtained for HOPG as a reference sample (Table 1 and Fig. 5), we have to take into account that HOPG, a mosaic crystal, possesses much larger domain sizes (typically from several to a few tens of micrometers) [78] and hence a much lower density of grain boundaries in its lattice. Because grain boundaries are highly reactive sites and generally act as scattering centers for the charge carriers [64], the reactivity of the HG1500-2700 film should be higher than that of HOPG (Fig. 5); similarly, its electrical 
conductivity should remain below that of HOPG and, a fortiori, below that of single-crystal graphite (Table 1). Consequently, further significant improvement in the electrical conductivity of the films should only be possible if we are able to decrease the density of grain boundaries. Since we cannot expect the annihilation of the grain boundaries even at graphitization temperatures higher than those employed here [76], a different approach must be adopted. Because the grain boundaries are assumed to originate from the edges of the individual reduced graphene oxide sheets, using much larger sheets in the preparation of the films could offer a way out. Aqueous dispersions of graphene oxide sheets with lateral sizes up to $50 \mu \mathrm{m}$ have been reported and could be used to this end [79]. (5) The coalescence of the sheets to yield continuous polycrystalline layers should imply a very high mobility of the carbon atoms involved in the process, particularly those at the sheet edges. It is thus conceivable that some of these atoms can go astray and end up trapped as interstitials in-between the graphene layers (Fig. 7(f)). The process of sheet coalescence could also be invoked to explain some a priori unexpected trends in the experimental results, for instance, the evolution of the doo2 parameter (Table 1). The onset of coalescence, which appeared to take place at the lowest temperatures of the second annealing step (Fig. 6), could disturb the stacking of the sheets on a local scale before interlayer consolidation is eventually achieved at the highest graphitization temperatures. This mechanism would account for the transitional increase in the d002 parameter of the films graphitized at 1800 and $2100{ }^{\circ} \mathrm{C}$ observed during the second annealing step (Table 1 ).

\section{Conclusions}

A very high level of structural restoration has been achieved for reduced graphene oxide sheets assembled into free-standing, paper-like films by graphitization, yielding thin films that approach the characteristics of highly oriented graphites. As a result, the electrical conductivity of the graphitized films reached values as high as $577,000 \mathrm{~S} \mathrm{~m}^{-1}$, which are by far the largest reported to date for any material derived from graphene oxide and about one quarter of the value measured for pristine single-crystal graphite, taken as the ultimate upper limit for graphitic systems. Extensive characterization of the films indicated that there are two main stages of transformation during their graphitization: (i) full removal of residual oxygen functional groups from the reduced graphene oxide sheets and concomitant generation of atomic vacancies (first annealing step, $1500{ }^{\circ} \mathrm{C}$ ), and (ii) annihilation of the vacancies and coalescence of adjacent overlapping sheets to yield continuous polycrystalline layers (second annealing step, 1800-2700 ${ }^{\circ} \mathrm{C}$ ). The individual domains in the polycrystalline layers were seen to be essentially free of even point defects and their size (a few hundred nanometers) was mainly determined by the dimensions of the starting reduced graphene oxide sheets. As the domains themselves were defect-free, the prevailing type of defect within the layers was the grain boundaries separating neighboring domains, which were thought to govern many properties of the highly graphitized films. Finally, we envisage that the method proposed here can also be applied to fully restore the graphitic lattice of isolated, individual reduced graphene oxide sheets (as opposed to sheets assembled into films), provided that suitable substrates are used for the sheets. Because of its extreme heat resistance and the fact that it has already been shown to be an ideal substrate for pristine graphene, hexagonal boron nitride could be a good candidate to this end.

\section{Acknowledgements}

Financial support from the Spanish MICINN and MINECO (project MAT2011-26399) is gratefully acknowledged. R.R. thanks the receipt of a pre-doctoral contract (FPU) from the Spanish MECD.

Electronic Supplementary Material. Further details on XPS analyses of the samples and Raman spectra of samples prepared by one-step annealing are available in the online version for this article at (to be inserted by the publisher). 


\section{References}

[1] Geim, A.K.; Novoselov, K.S. The rise of graphene. Nature Mater. 2007, 6, 183-191.

[2] Geim, A.K. Graphene: status and prospects. Science 2009, 324, 1530-1534.

[3] Schwierz, F. Graphene transistors. Nature Nanotech. 2010, 5, 487-496.

[4] Luo, B.; Liu, S.; Zhi, L. Chemical approaches toward graphene-based nanomaterials and their applications in energy-related areas. Small 2012, 8, 630-646.

[5] Liu, Y.; Dong, X.; Chen, P. Biological and chemical sensors based on graphene materials. Chem. Soc. Rev. 2012, 41, 2283-2307.

[6] Akhavan, O.; Ghaderi, E.; Rahighi, R. Toward Single-DNA Electrochemical Biosensing by Graphene Nanowalls. ACS Nano 2012, 6, 2904-2916.

[7] Machado, B.F.; Serp, P. Graphene-based materials for catalysis. Catal. Sci. Technol. 2012, 2, 54-75.

[8] Feng, L.; Liu, Z. Graphene in biomedicine: opportunities and challenges. Nanomedicine 2011, 6, 317-324.

[9] Park, S.; Ruoff, R. S. Chemical methods for the production of graphenes. Nature Nanotech. 2009, 4, 217-224.

[10] Wei, D.C.; Liu, Y.Q. Controllable synthesis of graphene and its applications. Adv. Mater. 2010, 22, 3225-3241.

[11] Guo, S.; Dong, S. Graphene nanosheet: synthesis, molecular engineering, thin film, hybrids, and energy and analytical applications. Chem. Soc. Rev. 2011, 40, 2644-2672.

[12] Dreyer, D.S.; Park, S.; Bielawski, C.W.; Ruoff, R.S. The chemistry of graphene oxide. Chem. Soc. Rev. 2010, 39, 228-240.

[13] Esfandiar, A.; Akhavan, O.; Irajizad, A. Melatonin as a powerful bio-oxidant for reduction of graphene oxide. $J$. Mater. Chem. 2011, 21, 10907-10914.

[14] Mao, S.; Pu, H.; Chen, J. Graphene oxide and its reduction: modeling and experimental progress. RSC Advances 2012, 2, 2643-2662.

[15] Pei, S.; Cheng, H.-M. The reduction of graphene oxide. Carbon 2012, 50, 3210-3228.
[16] Akhavan, O.; Ghaderi, E. Escherichia coli bacteria reduced graphene oxide to bactericidal graphene in a self-limiting manner. Carbon 2012, 50, 1853-1860.

[17] Akhavan, O.; Kalaee, M.; Alavi, Z. S.; Ghiasi, S. M. A, Esfandiar, A. Increasing the antioxidant activity of green tea polyphenols in the presence of iron for the reduction of graphene oxide Carbon 2012, 50, 3015-3025.

[18] Compton, O.C.; Nguyen, S.T. Graphene oxide, highly reduced graphene oxide, and graphene: versatile building blocks for carbon-based materials. Small 2010, 6, 711-723.

[19] Eda, G; Chhowalla, M. Chemically derived graphene oxide: towards large-area thin-film electronics and optoelectronics. Adv. Mater. 2010, 22, 2392-2415.

[20] Gómez-Navarro, C.; Meyer, J.C.; Sundaram, R.S.; Chuvilin, A.; Kurasch, S.; Burghard, M.; Kern, K.; Kaiser, U. Atomic structure of reduced graphene oxide. Nano Lett. 2010, 10, 1144-1148.

[21] Erickson, K.; Erni, R.; Lee, Z.; Alem, N.; Gannett, W.; Zettl, A. Determination of the local chemical structure of graphene oxide and reduced graphene oxide. Adv. Mater. 2010, 22, 4467-4472.

[22 ] Gómez-Navarro, C.; Weitz, R.T.; Bittner, A.M.; Scolari, M.; Mews, A.; Burghard, M.; Kern, K. Electronic transport properties of individual chemically reduced graphene oxide sheets. Nano Lett. 2007, 7, 3499-3503.

[23] Mattevi, C.; Eda, G.; Agnoli, S.; Miller, S.; Mkhoyan, K.A.; Celik, O.; Mastrogiovanni, D.; Granozzi, G.; Garfunkel, E.; Chhowalla, M. Evolution of electrical, chemical, and structural properties of transparent and conducting chemically derived graphene thin films. $A d v$. Funct. Mater. 2009, 19, 2577-2583.

[24] López, V.; Sundaram, R.S.; Gómez-Navarro, C.; Olea, D.; Burghard, M.; Gómez-Herrero, J.; Zamora, F.; Kern, K. Chemical vapor deposition repair of graphene oxide: a route to highly-conductive graphene monolayers. Adv. Mater. 2009, 21, 4683-4686.

[25] Dai, B.; Fu, L.; Liao, L.; Liu, N.; Yan, K.; Chen, Y.; Liu, Z. High-quality single-layer graphene via reparative reduction of graphene oxide. Nano Res. 2011, 4, 434-439. 
[26] Cheng, M.; Yang, R.; Zhang, L.; Shi, Z.; Yang, W.; Wang, D.; Xie, G.; Shi, D.; Zhang, G. Restoration of graphene from graphene oxide by defect repair. Carbon 2012, 50, 2581-2587.

[27] Kholmanov, I.N.; Edgeworth, J.; Cavaliere, E.; Gavioli, L.; Magnuson, C.; Ruoff, R.S. Healing of structural defects in the topmost layer of graphite by chemical vapor deposition. Adv. Mater. 2011, 23, 1675-1678.

[28] Matuyama, E. Pyrolysis of graphitic acid. J. Phys. Chem. 1954, 58, 215-219.

[29] Maire, J.; Colas, H.; Maillard, P. Membranes de carbone et de graphite et leurs propietes. Carbon 1968, 6 , 555-560.

[30] Toyoda, S.; Yamakawa, T.; Kobayashi, K.; Yamada, Y. Anisotropy of g-value in a graphitized carbon film. Carbon 1972, 10, 646-647.

[31] Matsuo, Y.; Sugie, Y. Preparation, structure and electrochemical property of pyrolytic carbon from graphite oxide. Carbon 1998, 36, 301-303.

[32] Matsuo, Y.; Sugie, Y. Pyrolytic carbon from graphite oxide as an anode of lithium-ion cells in $1 \mathrm{M} \mathrm{LiClO}_{4}$ propylene carbonate solution. Electrochem. Solid-State Lett. 1998, 1, 204-206.

[33] Matsuo, Y.; Sugie, Y. Electrochemical lithiation of carbon prepared from pyrolysis of graphite oxide. $J$. Electrochem. Soc. 1999, 146, 2011-2014.

[34] Yang, D.; Velamakanni, A.; Bozoklu, G.; Park, S.; Stoller, M.; Piner, R.D.; Stankovich, S.; Jung, I.; Field, D.A.; Ventrice Jr, C.A. et al. Chemical analysis of graphene oxide films after heat and chemical treatments by X-ray photoelectron and Micro-Raman spectroscopy. Carbon 2009, 47, 145-152.

[35] Akhavan, O. The effect of heat treatment on formation of graphene thin films from graphene oxide nanosheets. Carbon 2010, 48, 509-519.

[36] Chen, C.-M.; Huang, J.-Q.; Zhang, Q.; Gong, W.-Z.; Yang, Q.-H.; Wang, M.-Z.; Yang, Y.-G. Annealing a graphene oxide film to produce a free standing high conductive graphene film. Carbon 2012, 50, 659-667.
[37] Oberlin, A. Carbonization and graphitization. Carbon 1984, 22, 521-541.

[38] Long, D.; Li, W.; Qiao, W.; Miyawaki, J.; Yoon, S.-H.; Mochida, I.; Ling, L. Graphitization behaviour of chemically derived graphene sheets. Nanoscale 2011, 3, 3652-3656.

[39] Ghosh, T.; Biswas, C.; Oh, J.; Arabale, G.; Hwang, T.; Luong, N.D.; Jin, M.; Lee, Y.H.; Nam, J.-D. Solution-processed graphite membrane from reassembled graphene oxide. Chem. Mater. 2011, 24, 594-599.

[40] Abouimrane, A.; Compton, O.C.; Amine, K.; Nguyen, S.T. Non-annealed graphene paper as a binder-free anode for lithium-ion batteries. J. Phys. Chem. C 2010, 114, 12800-12804.

[41] Liang, J.; Huang, Y.; Oh, J.; Kozlov, M.; Sui, D.; Fang, S.; Baughman, R.H.; Ma, Y.; Chen, Y. Electromechanical actuators based on graphene and graphene/Fe3O4 hybrid paper. Adv. Funct. Mater. 2011, 21, 3778-3784.

[42] Zhang, L.L.; Zhao, X.; Stoller, M.D.; Zhu, Y.; Ji, H.; Murali, S.; Wu, Y.; Perales, S.; Clevenger, B.; Ruoff, R.S. Highly conductive and porous activated reduced graphene oxide films for high-power supercapacitors. Nano Lett. 2012, $12,1806-1812$.

[43] Gao, H.; Wang, Y.; Xiao, F.; Ching, C.B.; Duan, H. Growth of copper nanocubes on graphene paper as free-standing electrodes for direct hydrazine fuel cells. $J$. Phys. Chem. C 2012, 116, 7719-7725.

[44] Hummers, W.S.; Offeman, R.E. Preparation of graphitic oxide. J. Am. Chem. Soc. 1958, 80, 1339-1339.

[45] Paredes, J.I.; Villar-Rodil, S.; Solís-Fernández, P.; Martínez-Alonso, A.; Tascón, J.M.D. Atomic force and scanning tunneling microscopy imaging of graphene nanosheets derived from graphite oxide. Langmuir 2009, 25, 5957-5968.

[46] Fernández-Merino, M.J.; Guardia, L.; Paredes, J.I.; Villar-Rodil, S.; Solís-Fernández, P.; Martínez-Alonso, A.; Tascón, J.M.D. Vitamin $\mathrm{C}$ is an ideal substitute for hydrazine in the reduction of graphene oxide suspensions. $J$. Phys. Chem. C 2010, 114, 6426-6432. 
[47] Li, D.; Müller, M.B.; Gilje, S.; Kaner, R.B.; Wallace, G.G. Processable aqueous dispersions of graphene nanosheets. Nature Nanotech. 2008, 3, 101-105.

[48] Paci, J.T.; Belytschko, T.; Schatz, G.C. Computational studies of the structure, behavior upon heating, and mechanical properties of graphite oxide. J. Phys. Chem. C 2007, 111, 18099-18111.

[49] Bagri, A.; Mattevi, C.; Acik, M.; Chabal, Y.J.; Chhowalla, M.; Shenoy, V.B. Structural evolution during the reduction of chemically derived graphene oxide. Nature Chem. 2010, 2, 581-587.

[ 50 ] Solís-Fernández, P.; Rozada, R.; Paredes, J.I.; Villar-Rodil, S.; Fernández-Merino, M.J.; Guardia, L.; Martínez-Alonso, A.; Tascón, J.M.D. Chemical and microscopic analysis of graphene prepared by different reduction degrees of graphene oxide. J. Alloys Compd. 2012, 536S, S532-S537.

[51] Stankovich, S.; Dikin, D.A.; Piner, R.D.; Kohlhaas, K.A.; Kleinhammes, A.; Jia, Y.; Wu, Y.; Nguyen , S.T.; Ruoff, R.S. Synthesis of graphene-based nanosheets via chemical reduction of exfoliated graphite oxide. Carbon 2007, 45, 1558-1565.

[ 52 ] Kim, M.C.; Hwang, G.S.; Ruoff, R.S. Epoxide reduction with hydrazine on graphene: A first principles study. J. Chem. Phys. 2009, 131, 064704.

[53] Gao, X.; Jang, J.; Nagase, S. Hydrazine and thermal reduction of graphene oxide: reaction mechanisms, product structures, and reaction design. J. Phys. Chem. C 2009, 114, 832-842.

[54] Figueiredo, J.L.; Pereira, M.F.R.; Freitas, M.M.A.; Órfão, J.J.M. Modification of the surface chemistry of activated carbons. Carbon 1999, 37, 1379-1389.

[55] Chen, H.; Müller, M.B.; Gilmore, K.J.; Wallace, G.G.; Li, D. Mechanically strong, electrically conductive, and biocompatible graphene paper. Adv. Mater. 2008, 20, 3557-3561.

[56] Ferrari, A.C.; Robertson, J. Interpretation of Raman spectra of disordered and amorphous carbon. Phys. Rev. B 2000, 61, 14095-14107.
[57] Pimenta, M.A.; Dresselhaus, G.; Dresselhaus, M.S.; Cançado, L.G.; Jorio, A.; Saito, R. Studying disorder in graphite-based systems by Raman spectroscopy. Phys. Chem. Chem. Phys. 2007, 9, 1276-1290.

[58] Tuinstra, F.; Koenig, J.L. Raman spectrum of graphite. J. Chem. Phys. 1970, 53, 1126.

[59] Cançado, L.G.; Takai, K.; Enoki, T.; Endo, M.; Kim, Y.A.; Mizusaki, H.; Jorio, A.; Coelho, L.N.; Magalhães-Paniago, R.; Pimenta, M.A. General equation for the determination of the crystallite size La of nanographite by Raman spectroscopy. Appl. Phys. Lett. 2006, 88, 163106. [ 60 ] Malard, L.M.; Pimenta, M.A.; Dresselhaus, G.; Dresselhaus, M.S. Raman spectroscopy in graphene. Phys. Rep. 2009, 473, 51-87.

[61] Lahaye, J.; Ehrburger, P. Fundamental issues in control of carbon gasification reactivity; Kluwer Academic Publishers: Dordrecht, 1991.

[62] Cuesta, A.; Martínez-Alonso, A.; Tascón, J.M.D. Carbon reactivity in an oxygen plasma: a comparison with reactivity in molecular oxygen. Carbon 2001, 39, 1135-1146.

[63] Solís-Fernández, P.; Paredes, J.I.; Villar-Rodil, S.; Guardia, L.; Fernández-Merino, M.J.; Dobrik, G.; Biró, L.P.; Martínez-Alonso, A.; Tascón, J.M.D. Global and local oxidation behavior of reduced graphene oxide. J. Phys. Chem. C 2011, 115, 7956-7966.

[64] Banhart, F.; Kotakoski, J.; Krasheninnikov, A.V. Structural defects in graphene. ACS Nano 2010, 5, 26-41.

[65] Spain, I.L. Electronic transport properties of graphite, carbons, and related materials. Chem. Phys. Carbon 1981, 16, 119-304.

[66] Morelli, D.T.; Uher, C. $\mathrm{T}^{2}$ dependence of the in-plane resistivity of graphite at very low temperatures. Phys. Rev. B 1984, 30, 1080-1082.

[67] Nakajima, T.; Nakane, K.; Kawaguchi, M.; Watanabe, N. Preparation, structure and electrical conductivity of graphite intercalation compound with titanium fluoride. Carbon 1987, 25, 685-689.

[68] Hahn, J.R.; Kang, H. Vacancy and interstitial defects at graphite surfaces: Scanning tunneling microscopic study of 
the structure, electronic property, and yield for ion-induced defect creation. Phys. Rev. B 1999, 60, 6007-6017.

[69] Solís-Fernández, P.; Paredes, J.I.; Martínez-Alonso, A.; Tascón, J.M.D. New atomic-scale features in graphite surfaces treated in a dielectric barrier discharge plasma. Carbon 2008, 46, 1364-1367.

[70] Paredes, J.I.; Solís-Fernández, P.; Martinez-Alonso, A.; Tascón, J.M.D. Atomic vacancy engineering of graphitic surfaces: controlling the generation and harnessing the migration of the single vacancy. J. Phys. Chem. C 2009, 113, 10249-10255.

[71] Wong, H.S.; Durkan, C.; Chandrasekhar, N. Tailoring the local interaction between graphene layers in graphite at the atomic scale and above using scanning tunneling microscopy. ACS Nano 2009, 3, 3455-3462.

[72] Magonov, S.N.; Whangbo, M.-H. Surface Analysis with STM and AFM; VCH: Weinheim, 1996.

[73] Paredes, J.I.; Martínez-Alonso, A.; Tascón, J.M.D. Early stages of plasma oxidation of graphite: nanoscale physicochemical changes as detected by scanning probe microscopies. Langmuir 2002, 18, 4314-4323.

[74] Banhart, F. Irradiation effects in carbon nanostructures. Rep. Prog. Phys. 1999, 62, 1181.

[75] Barreiro, A.; Börrnert, F.; Rümmeli, M.H.; Büchner, B.; Vandersypen, L.M.K. Graphene at high bias: cracking, layer by layer sublimation, and fusing. Nano Lett. 2012, 12, 1873-1878.

[76] Kurasch, S.; Kotakoski, J.; Lehtinen, O.; Skákalová, V.; Smet, J.; Krill, C.E.; Krasheninnikov, A.V.; Kaiser, U. Atom-by-atom observation of grain boundary migration in graphene. Nano Lett. 2012, 12, 3168-3173.

[77] Simonis, P.; Goffaux, C.; Thiry, P.A.; Biró, L.P.; Lambin, Ph.; Meunier, V. STM study of a grain boundary in graphite. Surf. Sci. 2002, 511, 319-322.

[78] Ohler, M.; Sanchez del Rio, M.; Tuffanelli, A.; Gambaccini, M.; Taibi, A.; Fantini, A.; Pareschi, G. X-ray topographic determination of the granular structure in a graphite mosaic crystal: a three-dimensional reconstruction. J. Appl. Cryst. 2000, 33, 1023-1030.

[79] Dong, X.; Su, C.-Y.; Zhang, W.; Zhao, J.; Ling, Q.;
Huang, W.; Chen, P.; Li, L.-J. Ultra-large single-layer graphene obtained from solution chemical reduction and its electrical properties. Phys. Chem. Chem. Phys., 2010, 12, 2164-2169. 